\title{
Establishment of a Pancreatic Cancer Stem Cell Model Using the SW1990 Human Pancreatic Cancer Cell Line in Nude Mice
}

\author{
Yan Pan ${ }^{1,2 \&}$, Song Gao ${ }^{1,2 \&}$, Yong-Qiang Hua ${ }^{1,2}$, Lu-Ming Liu ${ }^{1,2 *}$
}

\begin{abstract}
Aim: To establish a pancreatic cancer stem cell model using human pancreatic cancer cells in nude mice to provide a platform for pancreatic cancer stem cell research. Materials and Methods: To establish pancreatic cancer xenografts using human pancreatic cancer cell line SW1990, nude mice were randomly divided into control and gemcitabine groups. When the tumor grew to a volume of $125 \mathrm{~mm}^{3}$, they treated with gemcitabine at a dose of $50 \mathrm{mg} / \mathrm{kg}$ by intraperitoneal injection of $0.2 \mathrm{ml}$ in the gemcitabine group, while the mice in control group were treated with the same volume of normal saline. Gemcitabine was given 2 times a week for 3 times. When the model was established, the proliferation of pancreatic cancer stem cells was observed by clone formation assay, and the protein and/or mRNA expression of pancreatic stem cell surface markers including CD24, CD44, CD133, ALDH, transcription factors containing Oct-4, Sox-2, Nanog and Gli, the key nuclear transcription factor in Sonic Hedgehog signaling pathway was detected by Western blot and/or RT-PCR to verify the reliability of this model. Results: This model is feasible and safe. During the establishment, no mice died and the weight of nude mice maintained above 16.5g. The clone forming ability in gemcitabine group was stronger than that of the control group $(p<0.01)$. In gemcitabine group, the protein expression of pancreatic cancer stem cell surface markers including CD44, and ALDH was up-regulated, the protein and mRNA expression of nuclear transcription factor including Oct-4, Sox-2 and Nanog was also significantly increased $(P<0.01)$. In addition, the protein expression of key nuclear transcription factor in Sonic Hedgehog signaling pathway, Gli-1, was significantly enhanced $(p<0.01)$. Conclusions: The pancreatic cancer stem cell model was successfully established using human pancreatic cancer cell line SW1990 in nude mice. Gemcitabine could enrich pancreatic cancer stem cells, simultaneously accompanied by the activation of Sonic Hedgehog signaling pathway.
\end{abstract}

Keywords: Pancreatic cancer stem cell model - gemcitabine - sw1990 - the transcription factors - the surface markers

Asian Pac J Cancer Prev, 16 (2), 437-442

\section{Introduction}

Pancreatic Cancer Stem Cell (PCSC) represents the tumor's subpopulation with the capacity to drive its growth, invasion and metastasis, and severely affects the clinical prognosis (Clarke et al., 2006; Dalerba et al., 2007). PCSC was discovered for the first time in 2007 ( $\mathrm{Li}$ et al., 2007). With the development of the research, more and more knowledge was achieved in the last decade. Emerging evidence has suggested that PCSC serve as a reservoir for chemoresistence, recurrence and tumor metastasis. It would benefit for the treatment and the patient survive through PCSC -directed therapies (Trumpp et al., 2008; Zhou et al., 2009). Therefore, the key step is to establish a scientific and feasible animal model for the study on mechanism of PCSC as well as the screening of effective medicines. However, so far, the acquisition of PCSC and the foundation of animal model haven't had an acknowledged solution.

After the overview of the literature, there are several ways to obtain PCSC: 1) Using cell sorting techniques:
The combinations of cell-surface markers can be selected by the Flow cytometry or the magnetic bead selection; 2) Selecting for a subpopulation of cells that efflux dyes by sorting (Wang et al., 2013; Asuthkar et al., 2013); 3) Adopting the CSC biological characteristics to separate PCSC, such as serum-free non-adherent culturing (Yin et al., 2011) and Chemotherapy intervention (Hermann et al., 2007).

By far, the animal model of PCSC is established by pancreatic cancer stem cells acquired from subcutaneous or orthotopic injection. There are four deficiencies in above three establishments of Animal Models: 1) PCSCs differentiate inevitably in vivo after inoculation; 2) Contamination of cell is likely happened in the Flow cytometry; 3) Large sample of animal models is difficult to acquire; 4) the established animal model does not match with the clinical phenomenon. Therefore, a practical and consistent animal models of PCSCs is an urgent problem.

Unlike the traditional methods, Jimeno (Jimeno et al., 2009) and his group explored in another way. In the direct xenograft model of pancreatic cancer, the treated

${ }^{I}$ Department of Integrative Oncology, Fudan University Shanghai Cancer Center, ${ }^{2}$ Department of Oncology, Shanghai Medical College, Fudan University, Shanghai, China ${ }^{\&}$ Equal contributors *For correspondence: Ilm1010@163.com 
tumor showed an enrichment in CSC markers such as CD24 and ALDH after induced with gemcitabine. It is popular applied in the recent researches on the stem cells and related medicines development (Gach et al., 2013).

Due to the main limitations like insufficient amount of tissue from human pancreatic tumor and the instability when the cells repeatedly passaged, we made a technical refinement. We also established pancreatic cancer stem cell model using human pancreatic cancer cell line SW1990 in nude mice. We detected the expression of surface markers and the nuclear transcription factor, aiming to further identify the reliability of the new animal model. The model would provide a platform for study on mechanism of PCSC as well as the anticancer drug discovery.

\section{Materials and Methods}

\section{Cell lines and cell culture}

The human pancreatic cancer cell lines SW1990 was purchased from American Type Culture Collection (ATCC) (Manassas, VA, USA), cultured in DMEM supplemented with $1 \%$ penicillin/ streptomycin, $10 \%$ fetal bovine serum (FBS), 2mML-glutamine and $1 \mathrm{mM}$ sodium pyruvate, at $37^{\circ} \mathrm{C}$ with $5 \% \mathrm{CO}_{2}$. The culture fluid was replaced once every 2 to 3 days and digested with $0.25 \%$ trypsin. The cells were passaged in 1:3 scale. The spherical clusters of cells grown under these conditions were named SW1990 CSCs.

\section{Treatment of mice bearing xenograft tumors with gemcitabine}

The SW1990 single cell suspension (concentration of $107 / \mathrm{ml}, 0.2 \mathrm{ml}$ per mice) was injected into the left armpit of the nude mice with $1 \mathrm{ml}$ syringe under aseptic conditions. A total of 42 nude mices were implanted. The local tumor lesion formed in a week. After the tumors' volume reach $125 \mathrm{~mm} 3$, the mice were divided into two groups randomly: (a) Experiment group: gemcitabine 50 $\mathrm{mg} / \mathrm{kg}$ twice weekly i.p. $(\mathrm{n}=35)$ and (b) Control group $(n=7)$ : peritoneal injection with the same amount of saline.. After tumors were treated for 3 times, 7 mices were randomly selected from the control tumors and gemcitabine-treated tumors. The mice were excised for analysis, so as to verify that pancreatic cancer stem cell properties of subcutaneously transplanted tumor cells (Figure 1).

Since the initiation of implantation, mice had been monitored daily for signs of toxicity and weighed twice per week. Tumor size was evaluated two times per week by caliper measurements using the following formula: tumor volume $=0.52 \times$ length $\times$ width 2 . Tumor volume growth curve and weight fluctuation curve of the nude mice had been depicted.

\section{Colony formation assay}

Colony-forming efficiency was determined by using a double-layer soft agar method. A total of 103 cells were plated in $0.35 \%$ agar over a layer of $0.5 \%$ agar containing DMEM and $10 \%$ FBS in 6-well plates. Cells were incubated for 14 days in a $\mathrm{CO} 2$ incubator, and colonies larger than $50 \mu \mathrm{m}$ were counted under an Olympus BX51 microscope.

\section{Quantitative real-time $R T-P C R$}

RNA was extracted from frozen tumor specimens by using an RNeasy mini kit (Invitrogen), and cDNA was synthesized by using the Superscript II system (Fermentas) in accordance with the manufacturer's instructions. Quantification of SOX-2, NANOG, OCT-4 and GLI1 mRNA was conducted using the SYBR Green RT-PCR kit (Fermentas) and ABI PRISM 7300 sequence detector (Applied BioSystems, Foster City, CA) according to manufacturers' instructions. Gene expression levels were calculated according to the following formula: $2-\triangle \mathrm{CT}$ $[\triangle \mathrm{CT}=\mathrm{Ct}$ (target)-Ct ( $\beta$-bactin)]. The amplification was performed using the following thermocycler parameters: initial denaturation at $95^{\circ} \mathrm{C}$ for $15 \mathrm{~min}$, followed by 30 cycles at $94^{\circ} \mathrm{C}$ for $30 \mathrm{sec}, 55^{\circ} \mathrm{C}$ for $30 \mathrm{sec}$ and $72^{\circ} \mathrm{C}$ for 45 seconds. The results were analyzed with melting curve analysis software (Dissociation Curve 1.0; Applied BioSystems) provided with the ABI PRISM 7300 sequence detector. The expression of mRNA was normalized to that of the reference gene, GAPDH. Relative quantification of mRNA within the samples was examined using the comparative $\mathrm{Ct}$ method. The details of primers are given in Table 1. Each experiment was repeated three times to confirm the results.

\section{Western blot analysis}

The protein concentration of the lysate was quantitated by BSA method. Equal amounts of lysate were loaded and separated by SDS-polyacrylamide gels. Then they were transferred onto nitrocellulose membranes. The membranes were blocked with 5\% non-fat milk powder in TBS for $1 \mathrm{~h}$ and probed with primary antibodies against CD24, CD44, ALDH1A1, CD133, Sox-2, Nanog, Oct-4 and Gli1. GAPDH was used as an internal control. After washed with TBS-T, the membrane was incubated with secondary horseradish peroxidase-coupled antibodies and visualized by using enhanced chemiluminescence.

\section{Statistical analysis}

The results were represented as the mean \pm standard deviation (SD). The statistical significance of differences was examined by Student's two-tailed t-test in two groups. The log-rank test was selected to compare the cumulative survival durations in different groups. Significant differences among groups were calculated at $p<0.05$. All data were analyzed with SPSS statistical software (SPSS Inc., Chicago, IL, USA).

\section{Results}

Gemcitabine-Induced Enrichment for Human SW1990 PCSC

A total number of 14 Human SW1990 cell line xenografts were established, 7 from gemcitabine group and 7 from control group. The average weight of the tumor tissue in control group was $0.022 \pm 0.010 \mathrm{~g}$ while the gemcitabine-treated group was $0.080 \pm 0.123 \mathrm{~g}(\mathrm{t}$ test, $p=0.353$ ) (Table 2). The weight of the nude mice 
in gemcitabine group dropped significantly while the no mice died during the establishment of animal model. The average weight of had significant difference as shown in Table 3. (t test, $p=0.000$ ), respectively (Table 2 ).

The identification of Human SW1990 PCSC

After the gemcitabine induction, we identified the enrichment condition of PCSC in gemcitabine group by detecting the followings: 1) Human PCSC proliferation; 2) The altering expression of cell surface markers; 3 ) Nuclear transcription factor. It helped to further identify the reliability of the pancreatic cancer stem cell model by using human pancreatic cancer cell line SW1990 in nude mice.

The Proliferative changes of PCSC after established modle

To observe the effects of PCSC on cell proliferation, we performed the cell viability assay and plate colony formation assay. The proliferation rate of PCSC was higher than that of the control cells. It formed 2.7 times colonies than the control cells with significant difference (Shown in Figure 2).

As showed in Figure 2, The gemcitabine group showed aggressive abilities of colony formation and proliferation in PCSCs. Colony formation of gemcitabine group was increased significantly than control group, $* p<0.05$. Asterisks indicate $p<0.05$ versus control group. Error bars represent standard deviations.

The alterative expression of Human SW1990 PCSC's cell surface markers and nuclear transcription factor

We detected the changes of markers on cancer stem cell surface and nuclear transcription factor by Western blot and/or RT-PCR to verify whether the PCSCs were enriched.

\section{Table 1. Real Time PCR Primer Base Sequence}

\begin{tabular}{|c|c|c|c|c|}
\hline \multicolumn{4}{|c|}{ The primer sequences } & Size \\
\hline \multicolumn{5}{|l|}{ SOX-2 } \\
\hline \multicolumn{5}{|c|}{ Forward 5'-GGGGGAAAGTAGTTTGCTGCCTCT-3' } \\
\hline \multicolumn{4}{|c|}{ Reverse 5'-TGCCGCCGCCGATGATTGTT-3' } & 135 \\
\hline \multicolumn{5}{|c|}{ OCT- 4} \\
\hline \multicolumn{5}{|c|}{ Forward 5'-GAGCAACTCCGATGGGGCCT-3' } \\
\hline \multirow{2}{*}{\multicolumn{5}{|c|}{ NANOG }} \\
\hline & & & & \\
\hline \multicolumn{5}{|c|}{ Forward 5'-GCAATGGTGTGACGCAGAAGGC-3' } \\
\hline Reverse 5'-' & GG & GTTGCTCCAC & TG-3' & 148 \\
\hline \multicolumn{5}{|c|}{ GLI1 } \\
\hline \multicolumn{5}{|c|}{ Forward 5'-AGGGAGTGCAGCCAATACAG-3' } \\
\hline Reverse 5'-1 & rG & GAGTTGATGT & & 171 \\
\hline \multicolumn{5}{|l|}{ GAPDH } \\
\hline \multicolumn{5}{|c|}{ Forward 5'-CAAGGTCATCCATGACAACTTTG -3' } \\
\hline \multicolumn{5}{|c|}{ Reverse 5'-GTCCACCACCCTGTTGCTGTAG -3' } \\
\hline \multicolumn{5}{|c|}{$\begin{array}{l}\text { Table 2. Tumor Weight and Body Weight of Nude Mice } \\
\text { After Induction with Gemcitabine }\end{array}$} \\
\hline & $\mathrm{n}$ & $\begin{array}{l}\text { Tumor weight } \\
(\mathrm{x} \pm \mathrm{SD})(\mathrm{g})\end{array}$ & $\begin{array}{r}\text { Bod } \\
(\mathrm{x} \pm\end{array}$ & eight \\
\hline Control & 7 & $0.080 \pm 0.123$ & & \\
\hline Gemcitabine & 7 & $0.022 \pm 0.010$ & 17. & \\
\hline P-value & & 0.353 & & \\
\hline
\end{tabular}

pancreatic cancer stem cell surface markers CD44, ALDH increased significantly

The results indicated that the protein expression of pancreatic cancer stem cell surface markers increased in different degree. the protein expression of CD44, ALDH is significantly up-regulated ( $\mathrm{t}$ test, $p=0.000$ ), while Membrane CD24 and CD133 did not significantly increased between groups and had no statistical variation compared with the control group $(p=0.064, p=0.053)$. ( Figure 3)

As showed in Figure3, The protein expression levels of PCSC surface markers between gemcitabine and control group were determined by Western blot analysis (left). The relative expression of protein revealed the differences between two groups (right). Asterisks indicate $p<0.05$ versus control group. Error bars represent standard deviations.

Up-regulation of nuclear transcription factor Sox-2 and Nanog

The results from western blot showed that nuclear transcription factor including Sox-2 and Nanog increased in different degrees $(p<0.05)$, which indicated that pancreatic cancer stem cells could be enriched by gemcitabine. In addition, the expression of key nuclear transcription factor, Gli-1, was significantly enhanced $(\mathrm{t}$

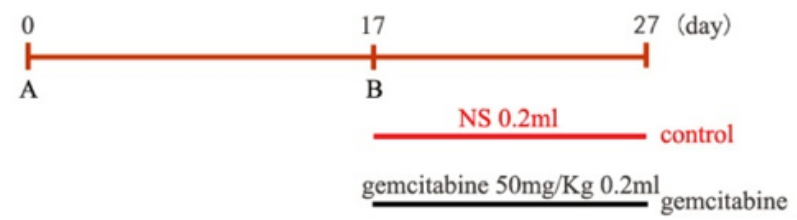

A: To establish pancreatic cancer xenografts using SW1990 cell line B: To divide the mice into control group and gemcitabine group randomly

Figure 1. The Flowchart of Establishment of Pancreatic Cancer Stem Cell Model Using Human Pancreatic Cancer Cell Line SW1990 in Nude Mice
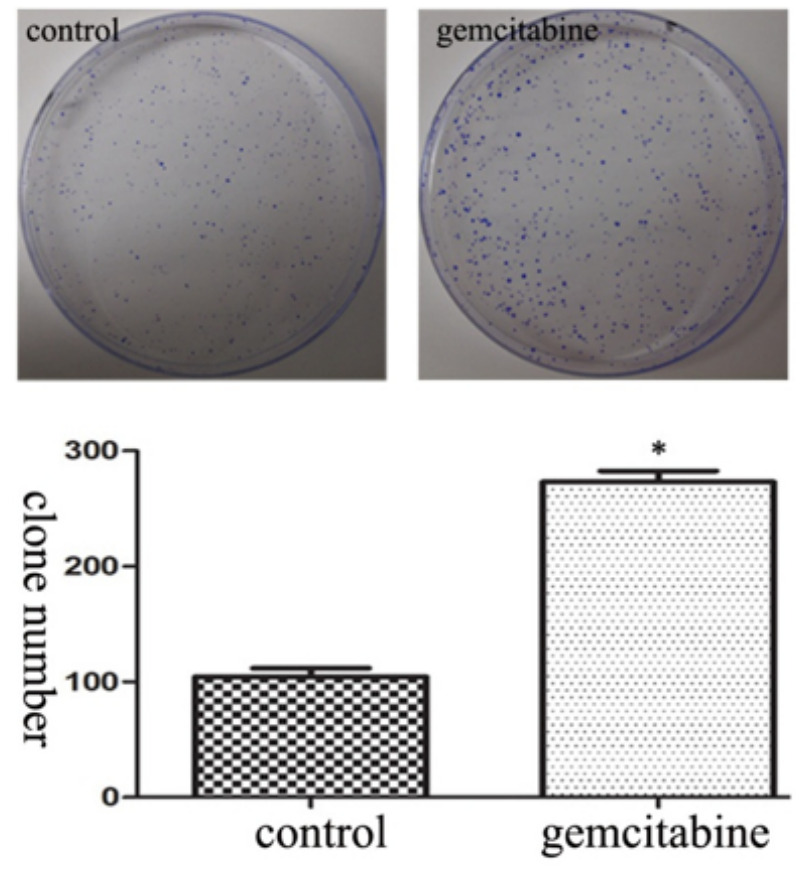

Figure 2. The Alteration of Colony Formation Ability 


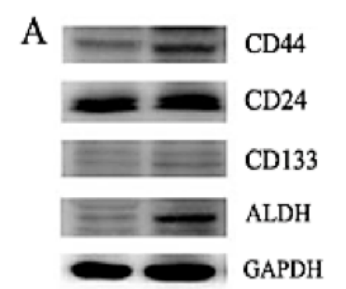

Control Gem

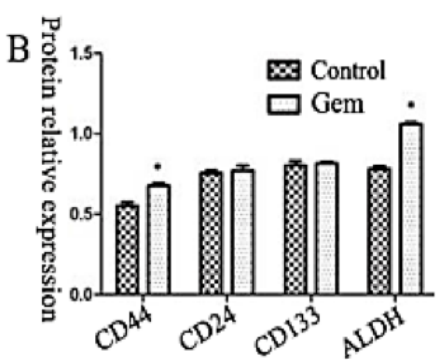

Figure 3. The Alterative Expression of Human SW1990 PCSC's Cell Surface Markers Including CD44, CD24, CD133 and ALDH

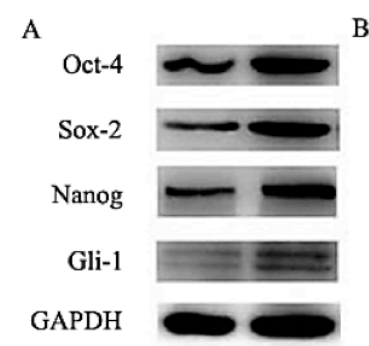

control Gem

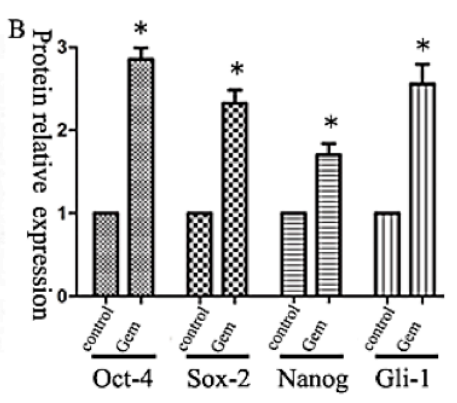

Figure 4. The Alteration of Protein Expression of Nuclear Transcription Factor Including Oct-4, Sox-2 and Nanog and Nuclear Transcription Factor of SHH Signaling Pathway, Gli-1, *p $<0.05$

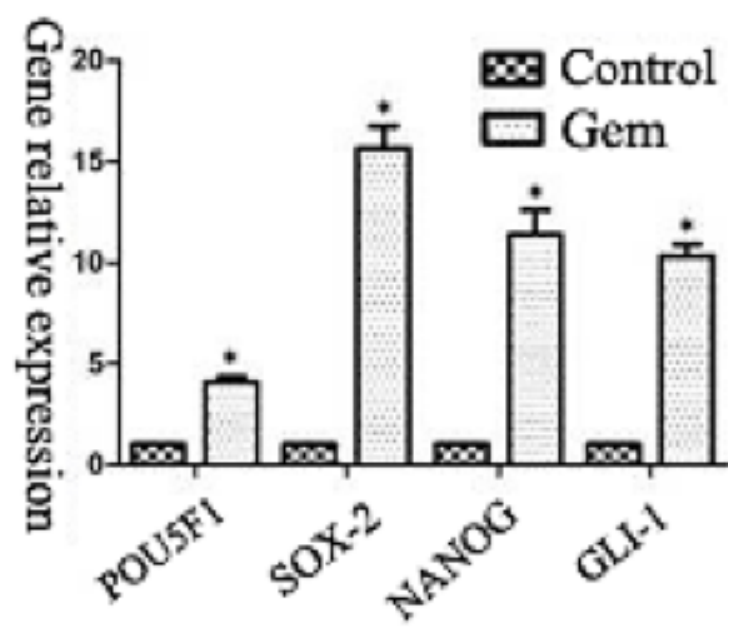

Figure 5. The mRNA Expression of Nuclear Transcription Factor Including Oct-4, Sox-2 and Nanog and Nuclear Transcription Factor of SHH Signaling Pathway, Gli-1, *p<0.05

test, $p=0.008$ ). (Figure 4)

As showed in Figure 4, The protein expression levels of nuclear transcription factor including Oct-4, Sox-2 and Nanog and nuclear transcription factor of SHH signaling pathway, Gli-1between gemcitabine and control group were determined by Western blot analysis (left). The relative expression of protein revealed the differences between two groups (right). Asterisks indicate $p<0.05$ versus control group. Error bars represent standard deviations.

The results of real-time RT-PCR demonstrated that the transcription of SOX-2, Oct-4 and Nanog mRNA were effectively increased. There has been a 16 folds increase in the mRNA expression of SOX-2 compared with control group ( $p=0.000$ ); besides, the mRNA expression of key nuclear transcription factor, Gli-1, was also significantly enhanced by 10 -fold $(p=0.001)$. (Figure 5)

As indicated in Figure 5, Real-time RT-PCR demonstrated that the mRNA expression of Oct-4, Sox-2, Nanog and Gli-1 were significantly increased respectively in Gemcitabine group compared with Control one. Asterisks indicate $p<0.05$ versus control group. Error bars represent standard deviations.

After gemcitabine intervention, the surviving SW1990 cells have a stronger proliferative capacity, along with the up-regulated mRNA expression of pancreatic cancer stem cell surface markers (CD44, ALDH, nuclear transcription factor containing Sox-2, Nanog). After induction with gemcitabine, treated tumor showed an increasing amount of human SW1990 pancreatic cancer stem cells, which means that human SW1990 PCSC were enriched.

\section{Discussion}

Pancreatic cancer is a highly malignant tumor with increasing incidence and mortality (Canyilmaz et al., 2013). From a theoretical perspective of CSC, tumor is a kind of stem cell disease (Hermann et al., 2007). CSC are a phenotypically-distinct population of cells that are functionally defined by their ability to form tumors, selfrenew, and differentiate, which served as a reservoir for disease recurrence and the origin of metastatic growth. Conventional cancer therapies, such as chemotherapy or radiation, can efficiently eradicate the majority of malignant cells within neoplastic lesions. However, increasing evidence showed that these regimens frequently fail to eliminate a minor subpopulation of resistant tumor cells that are malignant potential and dormant (Trumpp et al., 2008; Zhou et al., 2009). To date, research increasingly suggests that human pancreatic ductal adenocarcinoma (PDAC) contains a CSC. In clinical samples from patients, the presence of CSC or cells with potential of malignant transformation in tumor was associated with worse overall survival (Van et al., 2012; Kim et al., 2012).

The Definition and Biological Characteristics of Pancreatic Cancer Stem Cells (PCSC), the Correlationship between Clinical Prognosis and PCSC: Pancreatic Cancer Stem Cells (PCSC), a minor subpopulation of pancreatic cancer cells that have the potentiality of self-renewal and multi-directional differentiation. $\mathrm{Li}$ and his group found for the first a subpopulation of pancreatic cancer cells exists with markedly enhanced tumorigenic potential and stem pancreatic cell properties (Li et al., 2007), then Hermannn and his team subsequently also isolated the PCSC.

PCSC possesses five biological characteristics, including self-renew, multi-directional differentiation, high tumorigenicity, high metastasis and enhanced drugresistance. Van (Van et al., 2012) found for the first time that human pancreatic ductal adenocarcinoma (PDAC) contains a side population, which displaying CSCassociated activity and have a higher sphere-forming 
capacity than the main population cells. When culturing in serum-containing medium, these tumor spheres began to differentiate into normal pancreatic cancer cells (Pan et al., 2012). Li (Li et al., 2007) isolated Pancreatic cancer stem cells with the CD44+CD24+ESA+ phenotype by Flow cytometry, which had a 100-fold increased tumorigenic potential compared with non-tumorigenic cancer cells, although with a little percentage $0.2-0.8 \%$ of the total amount of pancreatic cancer cells. $50 \%$ of animals formed tumors, injected with as few as 100 CD44+ CD24+ ESA+ cells, which were histologically indistinguishable from the human tumors from which they originated. The pancreatic cancer cells expressing the cell surface markers CD44, CD24, and ESA had at least a 100 -fold increased tumorigenic potential compared with non-tumorigenic cells. Hermann (Hermann et al., 2007) isolated CD133+cells from freshly resected tumors by magnetic bead selection, and found that CD133+cells showed dramatic drug resistance to gemcitabine compared to autologous CD133-cells. In the invasive front of pancreatic tumors, a distinct subpopulation of CD133+CXCR4+cancer stem cells was identified that determines the metastatic phenotype of the individual tumor. Then they performed a study using continuous pharmacological inhibition of the CXCR4 receptor by AMD3100 for further evidence, with the result that AMD3100 treatment significantly reduced tumor metastasis.

Emerging evidence indicate that the tumor stem cell and the tumor poor prognosis is closely related. Drugresistant CSC, served as a reservoir for disease recurrence and the origin of metastatic growth, makes the extremely small part of highly tumorigenic CSC that remaining in the body to induce local recurrence and distant metastasis (Trumpp et al., 2008; Zhou et al., 2009). This hypothesis is confirmed by lots of clinical research, Van (Van et al., 2012) reported that the side population, more resistant to gemcitabine than the other tumor cells as examined in vivo, in human pancreatic ductal adenocarcinoma (PDAC) samples, which could be potential reason to recurrence of pancreatic cancer. Another study in 2012 found that up-regulated CD133 expression is in PDAC is related to poor prognosis (Kim et al., 2012). In addition, the presence of PCSC-related surface markers CD44+CD24+ (Ohara et al., 2013) or ALDH-expressing (Rasheed et al., 2010; Rasheed., 2012) in clinical samples from patients with resected pancreatic adenocarcinoma was associated with worse overall survival. Moreover, the high expressions of Oct4 and Nanog, which are crucial transcriptional regulators, in human pancreatic cancer tissues were found to indicate tumor progression and a worse prognostic value of patients ( $\mathrm{Lu}$ et al., 2013). Therefore, investigation of the potential role of PCSC in the genesis and progression of pancreatic cancer and the therapeutic strategy targeting the cancer stem cell have the certain positive sense to reduce the relapse following treatment, metastasis and prolong the survival time of patients, which becomes the new strategy for malignancy oncotherapy.

The Status of Acquisition of Stem Cell and Establishment of Animal Models: Although it has been 6 years since found of the PCSC, the acquisition of stem cell and establishment of animal models are still in the absence of a recognized standard.

Unlike the conventional methods, Jimeno (Jimeno et al., 2009) and his group explored in another way: after induced with gemcitabine, treated tumor showed an enrichment in CSC markers such as CD24 and ALDH, based on the direct xenograft model of pancreatic cancer. In the recent years, increasing researches on the stem cells and related medicines applied this method (Gach et al., 2013), which laid a good foundation for the further study on the biology activity of PCSCs' chemoresistance and metastasis, as well as screening and evaluation of medicines and effective elements targeting the CSCs. In this study, we employed a refined method to establish PCSC model. The modification is that we selected SW1990 cell line rather than the tissue from pancreatic cancer patients to set PCSC model. And the reasons why we chose SW1990 cell line and why we established this PCSC model are listed below.

We took SW1990 cell line as the object of this research because of its uniqueness, purity, stability and convenience. It was recorded that SW1990 cell line, a kind of epithelial cell, was cultured from the spleen metastases of a pancreatic cancer patient in 1978. In contrast, the tissue that obtained from pancreatic cancer patients in Jimeno's study was a bulk of several kinds of cells, thus it was more complicated to understand its specific role played in the mechanism of PCSC. Moreover, the aqusition of SW1990 is more convenient than that of pancreatic cancer tissue. It is known that $80 \%$ pancreatic cancer patients are at the advanced or terminal stage and it is unavailing for them to take a surgery, so obtaining cancer tissue is really hard. Secondly, look into the law of progress in pancreatic cancer, we can find that deterioration such as recurrence and metastasis occurs after chemotherapy. And pancreatic cancer stem cells represents the tumor's subpopulation with the capacity to drive invasion and metastasis, and they will be enriched after chemotherapy to affect the clinical prognosis, so we followed the rule of pancreatic cancer progression to set this PCSC model using gemcitabine. In other words, the model in the text is reasonable and feasible. Last but not least, we want to suggest the application of this PCSC model. It can be used to investigate the mechanisms involved in biological characteristics of PCSC, assess the effectiveness of cancer treatment, screen the agents targeting PCSC and so on.

The Evaluation of Transplanting Tumor Model on the Stem Cells Nude Mouse Subcutaneous Transplant: Based on all above method, we made a technical refinement and established pancreatic cancer stem cell model using human pancreatic cancer cell line SW1990 in nude mice, providing a platform for pancreatic cancer stem cell research.

During the establishment of animal model, nude mice in gemcitabine group, with a normal activity and food intake, maintained above $16.5 \mathrm{~g}$ despite their weight dropped, and no one died, indicating that our model is feasible and safe. $50 \mathrm{mg} / \mathrm{kg}$ is the endurant dose of gemcitabine for nude mice.

As pancreatic stem cell surface markers, CD44, CD24, 
CD133 and aldehyde dehydrogenase (ALDH) that have been commonly used for the identification of CSC in unrelated tissues. Moreover, the high expressions of Oct4, Sox-2, Nanog and Gli, the key nuclear transcription factor in Sonic Hedgehog signaling pathway, were found to indicate a worse prognostic value of patients. For this reason, we detected the altering expression of the above indexes after induction with gemcitabine, aiming to further identify the reliability of our established pancreatic cancer stem cell model using human pancreatic cancer cell line SW1990 in nude mice.

The results suggest that the clone forming ability in gemcitabine group was stronger than that of the control group; the protein expression of pancreatic cancer stem cell surface markers including CD44, and ALDH and nuclear transcription factor including Oct-4, Sox-2 and Nanog increased in varying degrees, which indicated that pancreatic cancer stem cells could be enriched by gemcitabine.

In addition, the protein and mRNA expression of key nuclear transcription factor, Gli-1, was both significantly enhanced, manifesting that the Sonic Hedgehog signaling pathway were activated. The Subsequent study on mechanism of PCSC could begin from the perspective of Sonic Hedgehog signaling pathway. Our method can be used for other cancer types such as breast cancer, colorectal cancer, hepatocellular carcinoma to evaluate or screen the efficiency of the drugs targeting cancer stem cells. However, due to the higher resection rate of the above cancer types than pancreatic cancer, this method can be a supplementary tool for CSC research.

\section{References}

AsuthkarS, Stepanova V,Lebedeva T, et al (2013). Multifunctional roles of urokinase plasminogen activator (UPA) in cancer stemness and chemoresistance of pancreatic cancer. $\mathrm{Mol}$ Biol Cell, 24, 2620-32.

Clarke MF, Dick JE, Dirks PB, et al (2006). Cancer stem cells-perspectives on current status and future directions: AACR Workshop on cancer stem cells. Cancer Res, 66, 9339-44.

Canyilmaz E, Serdar L, Uslu GH, et al (2013). Evaluation of prognostic factors and survival results in pancreatic carcinomas in Turkey. Asian Pac J Cancer Prev, 14, 6573-8.

Dalerba P, Cho RW, Clarke MF (2007). Cancer stem cells: models and concepts. Annu Rev Med, 58, 267-84.

Gach PC, Attayek PJ, Herrera G, et al (2013). Isolation and in vitro culture of rare cancer stem cells from patient-derived xenografts of pancreatic ductal adeno-carcinoma. Anal Chem, 85, 7271-78.

Hermann PC, Huber SL, Herrler T, et al (2007). Distinct populations of cancer stem cells determine tumor growth and metastatic activity in human pancreatic cancer. Cell Stem Cell, 1, 313-23.

Jimeno A, Feldmann G, Suarez-Gauthier A et al (2009). A direct pancreatic cancer xenograft model as a platform for cancer stem cell therapeutic development. Mol Cancer Ther, 8 , 310-4.

Kim HS, Yoo SY, Kim KT, et al (2012). Expression of the stem cell markers CD133 and nestin in pancreatic ductal adenocarcinoma and clinical relevance. Int J Clin Exp Pathol, 5, 754-61.

Li C, Heidt DG, Dalerba P et al (2007). Identification of pancreatic cancer stem cellsJ. Cancer Res, 67, 1030-7.

Lu Y, Zhu H, Shan H, et al (2013). Knockdown of Oct4 and Nanog expression inhibits the stemness of pancreatic cancer cells. Cancer Lett, 340, 113-23.

Ohara Y, Oda T, Sugano M, et al (2013). Histological and prognostic importance of CD44 (+)/CD24 (+)/Ep CAM $(+)$ expression in clinical pancreatic cancer. Cancer Sci, 104, 1127-34.

Pan Y, Hua YQ, Liu LM, et al (2012). Isolation and identification of pancreatic cancer stem cells from BxPC-3 cell line cultured in serum-free medium. China Cancer, 21, 441-5.

Rasheed ZA, Matsui W (2012). Biological and clinical relevance of stem cells in pancreatic adenocarcinoma. Gastroenterol Hepatol, 27, 15-8.

Rasheed ZA, Yang J, Wang Q, et al (2010). Prognostic significance of tumorigenic cells with mesenchymal features in pancreatic adenocarcinoma. Natl Cancer Inst, 102, 340-51.

Trumpp A, Wiestler OD (2008). Mechanisms of Disease: cancer stem cells--targeting the evil twin. Nat Clin Pract Oncol, 5, 337-47.

Van den Broeck A, Gremeaux L, Topal B, et al (2012). Human pancreatic adenocarcinoma contains a side population resistant to gemcitabine. BMC Cancer, 12, 354.

Wang X, Liu Q, Hou B, et al (2013). Concomitant targeting of multiple key transcription factors effectively disrupts cancer stem cells enriched in side population of human pancreatic cancer cells. PLoS One, 8, 73942.

Yin T, Wei H, Gou S, et al (2011). Cancer stem-like cells enriched in panc-1 spheres possess increased migration ability and resistance to gemcitabine. Int J Mol Sci, 12, 1595-604.

Zhou BB, Zhang H, Damelin M, et al (2009). Tumour-initiating cells: challenges and opportunities for anticancer drug discovery. Nat Rev Drug Discov, 8, 806-23. 